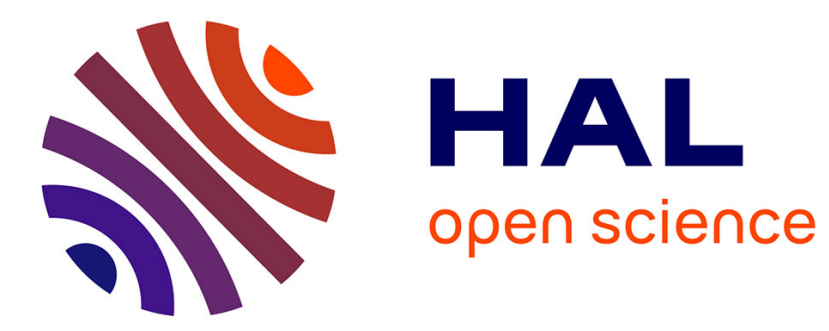

\title{
Aircraft Engine Blades-casing Contact Study
}

Isabelle Guilloteau, Etienne Arnoult, Bernard Peseux, Jean-Marc Berthillier

\section{To cite this version:}

Isabelle Guilloteau, Etienne Arnoult, Bernard Peseux, Jean-Marc Berthillier. Aircraft Engine Bladescasing Contact Study. 2nd Integrated Design and Manufacturing in Mechanical Engineering Conference (IMMD'98), May 1998, Compiègne, France. pp.35-42, 10.1007/978-94-015-9198-0_5 . hal01007473

\section{HAL Id: hal-01007473 \\ https://hal.science/hal-01007473}

Submitted on 7 Dec 2017

HAL is a multi-disciplinary open access archive for the deposit and dissemination of scientific research documents, whether they are published or not. The documents may come from teaching and research institutions in France or abroad, or from public or private research centers.
L'archive ouverte pluridisciplinaire HAL, est destinée au dépôt et à la diffusion de documents scientifiques de niveau recherche, publiés ou non, émanant des établissements d'enseignement et de recherche français ou étrangers, des laboratoires publics ou privés. 


\title{
AIRCRAFT ENGINE BLADES-CASING CONTACT STUDY
}

\author{
I. GUILLOTEAU, E. ARNOULT, B. PESEUX
}

Laboratoire de Mécanique et Matériaux, Division Structures, Ecole Centrale de Nantes, BP 92101, F-44321 NANTES Cedex 3, France M. BERTHILLIER

Département YLEA

SNECMA, F-77550 MOISSY-CRAMAYEL, France

\begin{abstract}
The present work aims at studying contact phenomena within aircraft engines. These events occur for instance when severe manoeuvrings are operated or when the engine bears a blade-off event. Two approaches are used simultaneously. The former is a numerical one and consists in a fine rotor/stator contact simulation. Various contactimpact algorithms and time integration schemes are tested on elementary cases. The Lagrange multiplier method appears to be the most appropriate one for dynamic studies. The latter approach is an experimental one and consists in a vibratory study of the mechanical coupling between the casing and the fan bladed disk so as to determine what parameters generate interaction. A simplified experimental test rig is used. Interaction is produced with selected casing and blade modes.
\end{abstract}

\section{Industrial Context}

During the qualification stage of a civil aircraft engine, a series of tests are carried out. One of these tests is a blade off test that demonstrates the engine capability of containing damage without catching fire and without failure of its mounting attachments when operated for at least 15 seconds, unless the resulting engine damage induces a self shutdown. The engineers need to predict the real loads applied to the engine structure in order to optimize the design stage. For this, a lot of numerical simulations are carried out but current software does not give adequate results, and in particular the finite element simulations involving contact. The aim of our work is to propose simplified models providing accurate results. The first part of this paper deals with the numerical simulation of contact, while the second part is an experimental study. 


\section{Algorithmic Aspects}

In order to build a simplified model of the dynamic contact phenomenon, classical contact-impact methods and time integration operators are studied.

\subsection{CONTACT METHODS}

Two parameters must be defined to characterize the contact conditions : the gap function $\mathrm{g}$, which represents the distance between the bodies in potential contact, and the contact pressure $\lambda$. Thus, the unilateral contact law is expressed as an impenetrability condition $(\mathrm{g} \geq 0)$, a compressive condition $(\lambda \leq 0)$, and a complementary condition ( $\mathrm{g} \lambda=0)$.

The discretized contact problem consists in finding the displacement vector $U(t)$, so that $M \ddot{U}+F^{\text {int }}(U, \dot{U})-F^{\text {ext }}+F^{\text {contact }}=0$ and $G(t)=G^{0}+Q U$, where $M$ is the mass matrix, $F^{\text {int }}$ is the vector of internal forces, $F^{\text {ext }}$ is the vector of external forces, $F^{\text {contact }}$ is the vector of contact forces, $\ddot{U}$ and $\dot{U}$ are the vectors of nodal accelerations and velocities. The gap functions have been adapted to the finite element method and are noted $G$ and $G^{0}$, vectors of current and initial gaps. $Q$ is a matrix deduced from the discretization of the contact entities.

The contact methods derive from the optimization study with constraints. The first one is the penalty method, adopted by commercial software of dynamic mechanics like LS-DYNA3D, PAMCRASH or RADIOSS. It consists in introducing artificial springs between the contact nodes. This method is easy to implement in a finite element code but admits body penetrations and induces high frequency oscillations. The problem becomes : find U so that

$$
M \ddot{U}+F^{\text {int }}(U, \dot{U})+\left[Q^{T} \alpha Q\right] U-F^{e x t}+Q^{T} \alpha G^{0}=0
$$

where the contact force vector is $\Lambda=\alpha \mathrm{G}$ and $\alpha$ is the penalty parameter matrix.

The second method is the Lagrange multiplier method, which has the advantage of enforcing the exact geometric contact condition but introduces additional variables, namely the contact forces. The problem is then to find the couple $(\mathrm{U}, \Lambda)$ so that

$$
\mathrm{MU}+\mathrm{F}^{\mathrm{int}}(\mathrm{U}, \dot{\mathrm{U}})-\mathrm{F}^{\mathrm{ext}}+\mathrm{Q}^{\mathrm{T}} \Lambda=0
$$

with the condition $G(t)=G^{0}+Q U=0$.

Another kind of method is a mix of the penalty functions and the Lagrange multipliers : for example, the augmented Lagrangian or the perturbed Lagrangian methods. We will not deal with these methods in the present paper.

\subsection{TIME INTEGRATION SCHEMES}

The scheme generally chosen for high velocity impact problems is an explicit one : the second-order accurate central difference method

$$
\mathrm{U}_{\mathrm{n}+1}=\mathrm{U}_{\mathrm{n}}+\Delta \mathrm{T} \dot{\mathrm{U}}_{\mathrm{n}+1 / 2}, \dot{\mathrm{U}}_{\mathrm{n}+1 / 2}=\dot{\mathrm{U}}_{\mathrm{n}-1 / 2}+\Delta \mathrm{T} \ddot{\mathrm{U}}_{\mathrm{n}}
$$

with $\Delta T=t_{n+1}-t_{n}=t_{n+1 / 2}-t_{n-1 / 2}$. 
This scheme is conditionally stable, i.e. the time step must be lower than a limit value, depending on the discretization of the structure : $\Delta \mathrm{T} \leq 2 / \mathrm{w}_{\max }$, where $\mathrm{w}_{\max }$ is the highest frequency component in the discretized system.

In the case of low velocities, an implicit scheme can be used. The most common one is the Newmark time integration scheme

$$
\begin{gathered}
\mathrm{U}_{\mathrm{n}+1}=\mathrm{U}_{\mathrm{n}}+\Delta \mathrm{T} \dot{\mathrm{U}}_{\mathrm{n}}+\Delta \mathrm{T}^{2}\left[(1 / 2-\beta) \ddot{\mathrm{U}}_{\mathrm{n}}+\beta \ddot{\mathrm{U}}_{\mathrm{n}+1}\right] \\
\dot{\mathrm{U}}_{\mathrm{n}+1}=\dot{\mathrm{U}}_{\mathrm{n}}+\Delta \mathrm{T}\left[(1-\gamma) \ddot{\mathrm{U}}_{\mathrm{n}}+\gamma \ddot{\mathrm{U}}_{\mathrm{n}+1}\right]
\end{gathered}
$$

where $\beta$ and $\gamma$ are the Newmark parameters. Integration is globally first-order accurate for $\gamma=1 / 2$ and a choice of $2 \beta \geq \gamma \geq 1 / 2$ leads to an unconditional stability. If $\beta=0$ and $\gamma=1 / 2$, the scheme corresponds to the central difference method.

\subsection{FORWARD INCREMENT LAGRANGE MULTIPLIER}

If the discretized contact problem is resolved using the central difference method for time integration and the Lagrange multiplier for contact, there appears a singularity : the contact forces $\Lambda_{n+1}$ have no influence on the displacements $U_{n+1}$ ! Carpenter et al. [3] propose an alternative formulation compatible with the explicit scheme : $M \ddot{U}_{n}+F^{\text {int }}\left(U_{n}, \dot{U}_{n}\right)-F_{n}^{e x t}+Q_{n+1}^{T} \Lambda_{n}=0, G_{n+1}=0$ (and not $G_{n}=0$ ).

The formulation leads to a predictor-corrector process $U_{n+1}=U_{n+1}^{*}+U_{n+1}^{c}$, where $\mathrm{U}_{\mathrm{n}+1}^{*}$ is the vector of nodal displacements calculated without contact. The vector $\mathrm{U}_{\mathrm{n}+1}^{\mathrm{c}}=-\Delta \mathrm{T}^{2} \mathrm{M}^{-1} \mathrm{Q}_{\mathrm{n}+1}^{\mathrm{T}} \Lambda_{\mathrm{n}}$ is a displacement correction due to contact and the force that permits us to impose the contact constraint is

$$
\Lambda_{n}=\left[\Delta T^{2} Q_{n+1} M^{-1} Q_{n+1}^{T}\right]^{-1}\left(Q_{n+1} U_{n+1}^{*}+G^{0}\right) \text {. }
$$

\section{Numerical Results}

The different methods of dynamic contact are tested on simple examples. The first one is an impact of two identical elastic rods, initially separated, as described in [3]. The methods used to resolve this problem are the penalty and the forward Lagrange multiplier methods, with an explicit time integration scheme. Figure 1 shows that the Lagrange multipliers give very accurate results compared to the penalty method.

If the penalty parameter is increased for a better precision of the results, the nodal penetration decreases but a lot of numerical oscillations appear in the velocity and contact force curves. Theoretically an infinite value must be used for the penalty parameter, but in practice, especially with an explicit time scheme, the contact stiffness should be of the same magnitude as the structure stiffness, otherwise the time step must be widely reduced. That is the reason why the results are not always satisfactory. 
With the Lagrange multiplier method, the results are accurate, and the explicit stable time step does not need decreasing [1]. Thus, in order to model the following examples, the Lagrange multiplier method is retained.

The second example deals with the impact of an elastic rod against a rigid wall, resolved using the Lagrange multiplier method with implicit and explicit time integration schemes and LS-DYNA3D, with the default contact values. The Newmark scheme with $(\beta=0.25 ; \gamma=0.55)$ does not give satisfactory results; there appears numerous oscillations in particular in the contact force curve [4]. The $(\beta=0.50 ; \gamma=0.55)$ implicit and the explicit schemes, coupled with the Lagrange multiplier method, give similar results, while the explicit-penalty method (LS-DYNA3D) induces a slight nodal penetration.
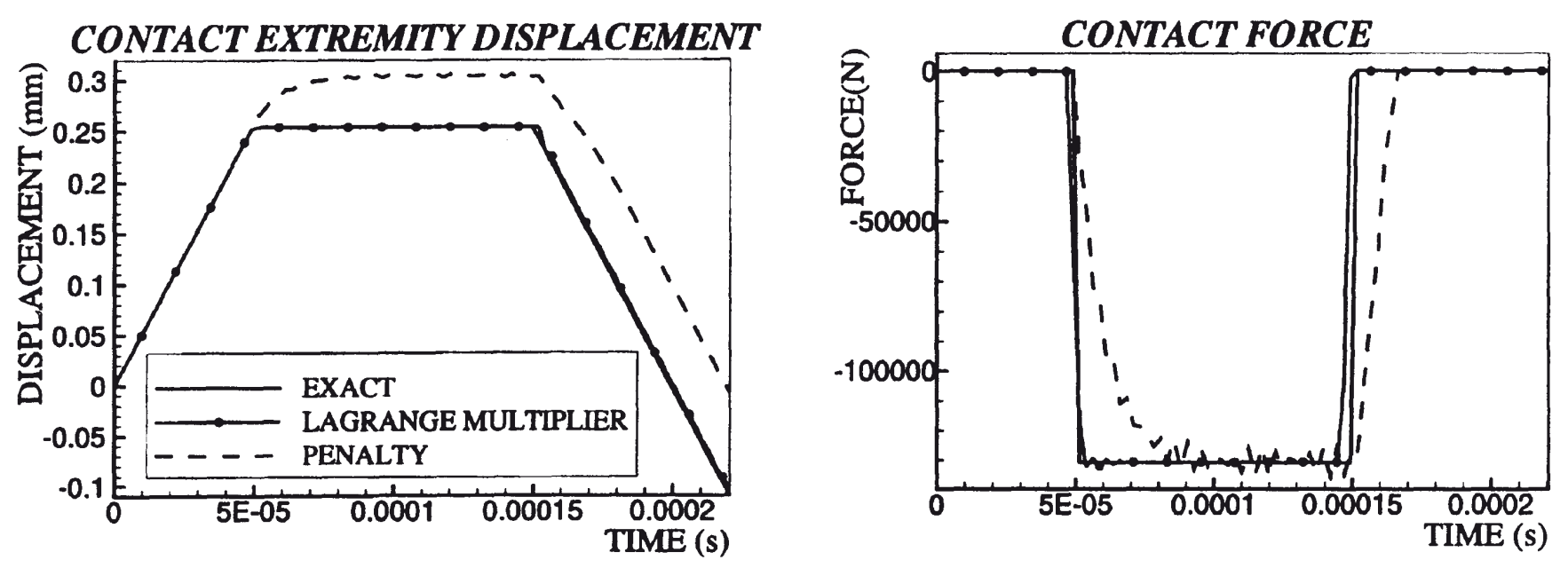

Figure 1. Impact of two identical elastic rods.

The last numerical test is the rebound, without friction, of a 2D rotational elastic beam against a rigid circle (cf. Figure 2). The steel beam describes an eccentric circle with the contact one and is subjected to a torque at its extremity on the hinge.
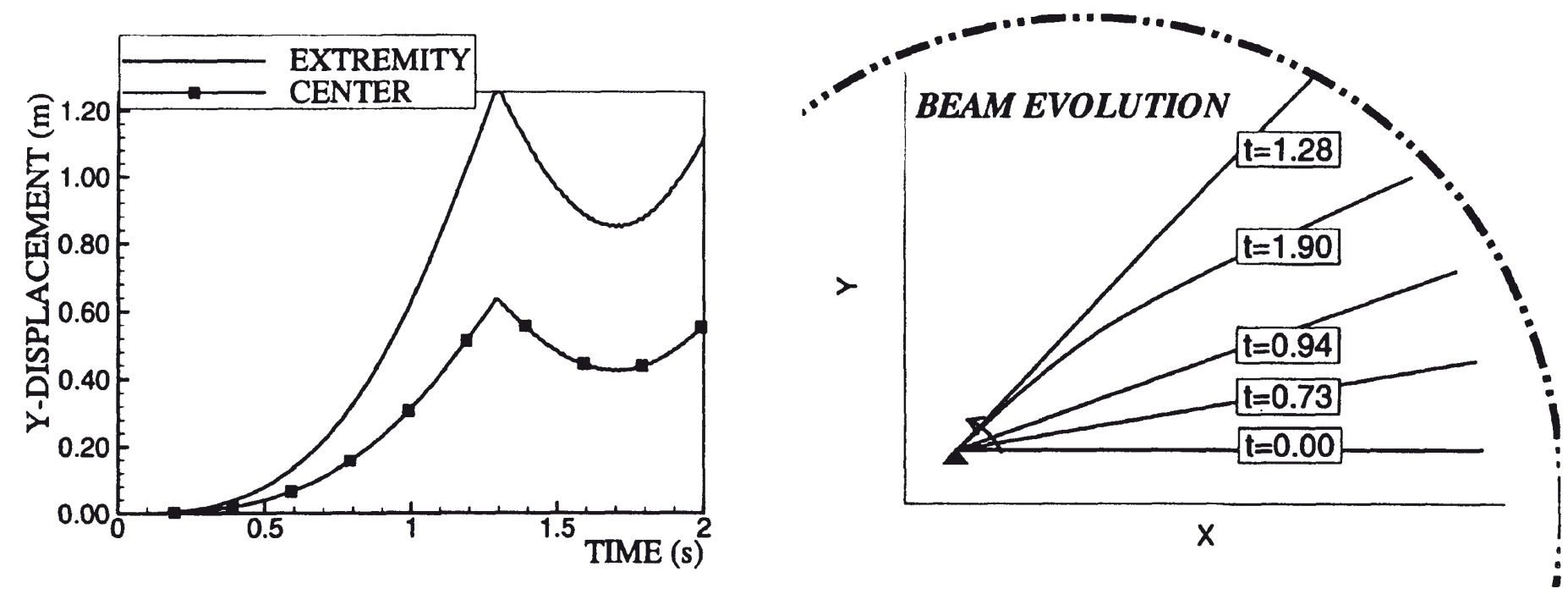

Figure 2. Beam rebound on a circular contact zone.

The next stage of our numerical study consists in modelling contact with a flexible axisymmetric surface. 


\section{Experimental Aspects}

\subsection{DYNAMIC INSTABILITY}

The experimental part of the study deals with vibratory interaction between the engine and the fan bladed disk. When an aircraft engine bears a blade loss, high unbalanced loads are generated. The bladed disk and the casing come into contact, shocking and rubbing each other. In the meantime the two structures are loaded over a huge frequency spectrum and numerous natural modes of vibration are excited. Some particular conditions are required to make the whole system come into resonance and be submitted to severe stresses. The dynamic instability can lead to the ruin of the engine. Special attention is therefore to be paid on the exact conditions which give rise to the instability.

Both the casing and the bladed disk are supposed to be axisymmetric structures. As a consequence, their natural modal shapes can be represented by the number of their nodal diameters $n$. If $f_{c}$ denotes the casing natural frequency for $n$ nodal diameters and $f_{b}$ the bladed disk natural frequency for the same $\mathrm{n}$ diameters mode, if $\mathrm{f}$ denotes the rotation frequency of the engine, then a condition for dynamic instability is given by [5]:

$$
f_{c}+f_{b}=n . f
$$

This equation means that waves in opposing directions are travelling both in the casing and the blades.

In order to avoid such a coincidence, engine designers are to respect important frequency constraints which usually lead to increasing the casing stiffness, weighting the engine down and making it clumsy. Other solutions have to be found.

\subsection{EXPERIMENTAL TEST RIG}

The purpose of the experimental approach is to examine more precisely the behaviour of the blades when rubbing on the casing, and validate numerical simulations. In a second part, the test rig will be used to determine which parameters are involved in the apparition of interaction, in addition to equation (7).

The test rig used is deliberately simplified (see Figure 3). It consists in a part of a casing of an aircraft engine bolted on a steel ring which is fixed on the table of a vertical lathe, and in a blade clamped in the turret. In this configuration, the rotating part is the casing, and no aerodynamic effect due to the rotation of blades has to be taken into account. The use of a single blade instead of a whole set is convenient to get rid of the problem of the direction of the travelling waves through the blades. The contact between the blade and the casing, as well as the lathe rotation, are controlled by the experimenter. The instrumentation is made of classical devices: accelerometers $(\mathrm{B} \& \mathrm{~K})$, amplifiers $(\mathrm{B} \& \mathrm{~K})$, spectral analyzer (HP), and is completed with a Laser Doppler Velocimeter (Polytec), useful for non-contact measurements on the rotating casing.

A first set of measurements was taken to characterize the test rig, especially to determine the natural frequencies and modal shapes of the casing. A finite element model including the casing and the steel ring was established and modified with respect to the experimental results. For a chosen rotation frequency of the lathe, interaction will 
be induce with a selected mode of the casing and a corresponding frequency of the blade.

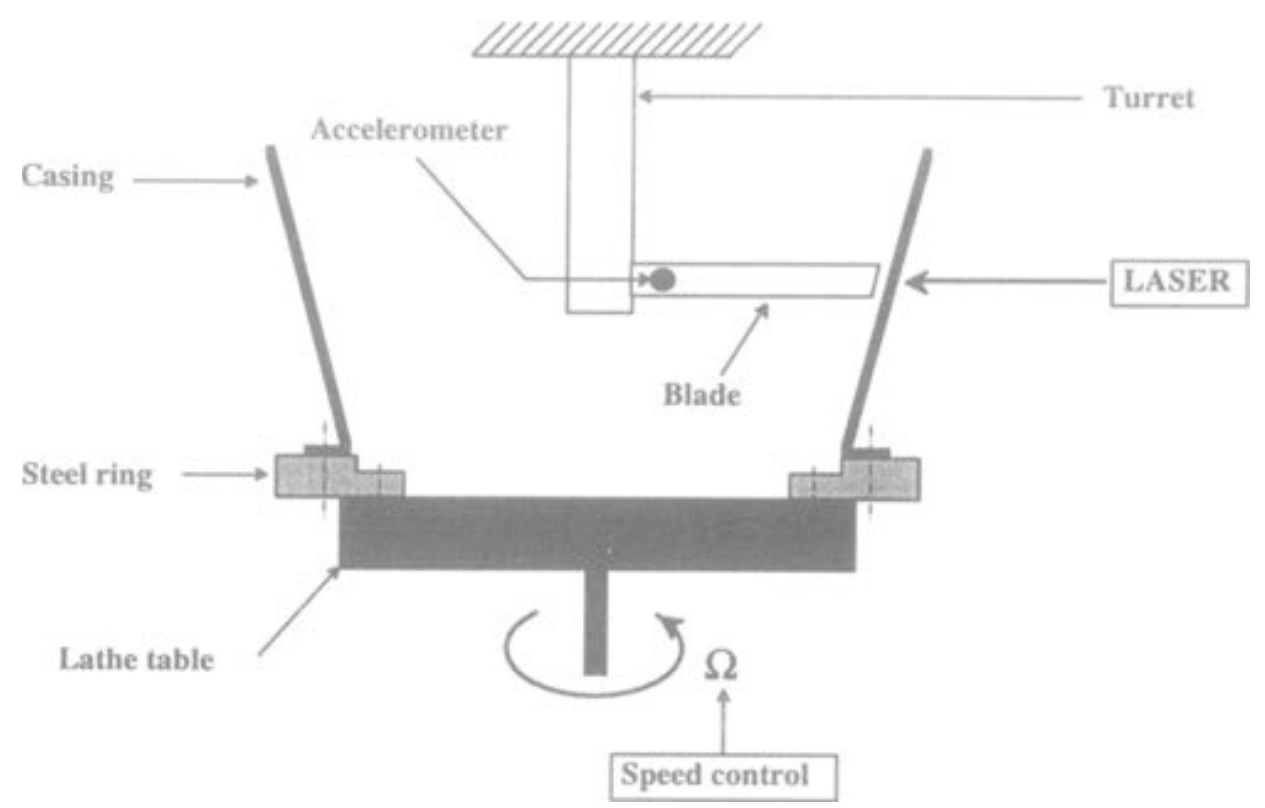

Figure 3. Experimental test rig.

\subsection{FINITE ELEMENT MODEL - MODAL ANALYSIS}

As a first approximation neglecting geometric and material defaults, the casing which looks like a frustrum of a cone is considered as an axisymmetric structure. The natural modes of this kind of structures have the particularity to be double ones: to each natural frequency correspond two different modal shapes. When the axisymmetry is perfect, and if $\theta$ denotes an angular parameter of the structure, the modal shapes in the radial direction $\operatorname{are} \cos (n \theta)$ and $\sin (n \theta)$, where $n$ stands for the number of nodal diameters. No special orientation is noticeable. In case of imperfections, the symmetry is broken and the system is said to be mistuned. Each natural frequency splits into two distinct ones, and the modal shapes are modified according to the importance of the unbalancement. Particular orientations appears [8].

The frequency response of the casing was recorded while the lathe was stopped, with a method using a small rotating mirror making the laser spot describe a circle on the inner side of the casing [2]. Once the identification of natural frequencies was complete (see Figure 4), time acquisitions were made to extract the modal shapes (see Figure 5). It appeared that the structure was mistuned both in frequency and time response, the biggest frequency split being obtained for the 4 nodal diameters mode (this is due to the way the steel ring was fixed on the lathe). The lowest modes were the most perturbed ones.

A first finite element model was made with SAMCEF supposing the perfect axisymmetry of the casing. A modal analysis carried out on the model clearly indicated that such an assumption was unacceptable. A fine geometrical study of the casing revealed that for a given altitude, the radius was not constant, the small variation, which never exceeds $1 \%$ being significant enough to disturb the modal behaviour. Another finite element model was then made including the geometric alterations. 
The new results are compared with the experimental results in Figure 6. The biggest differences are attributed to the imperfections of the casing embedding.

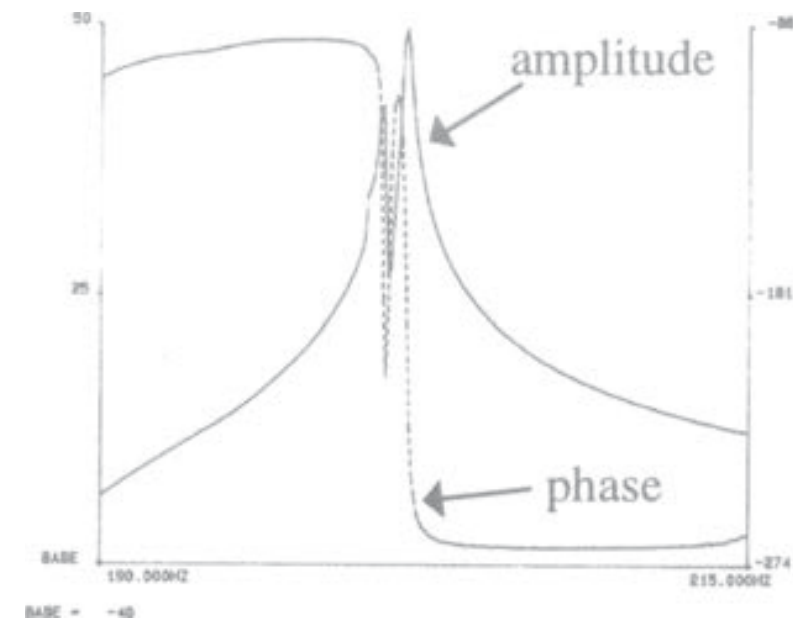

Figure 4. Casing frequency response.

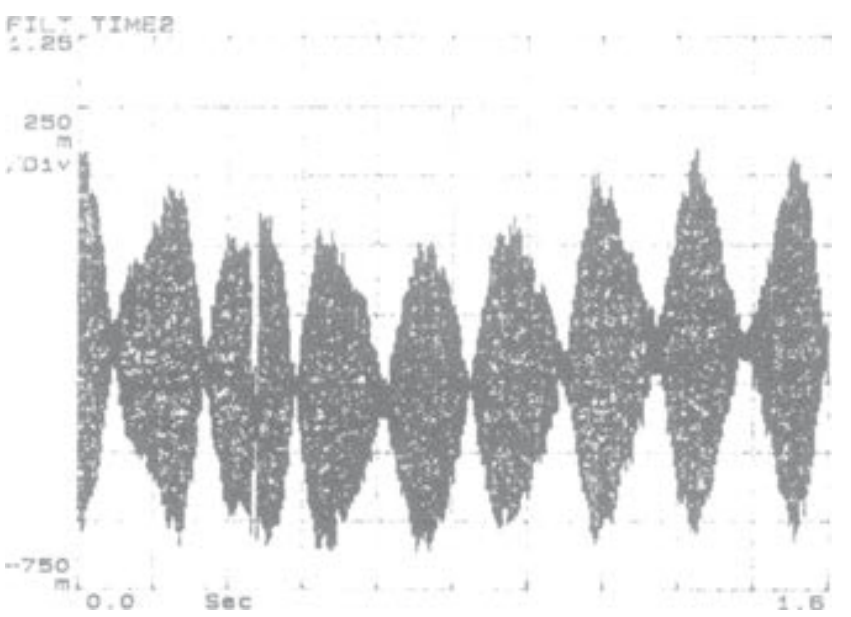

Figure 5. Example of modal shape.

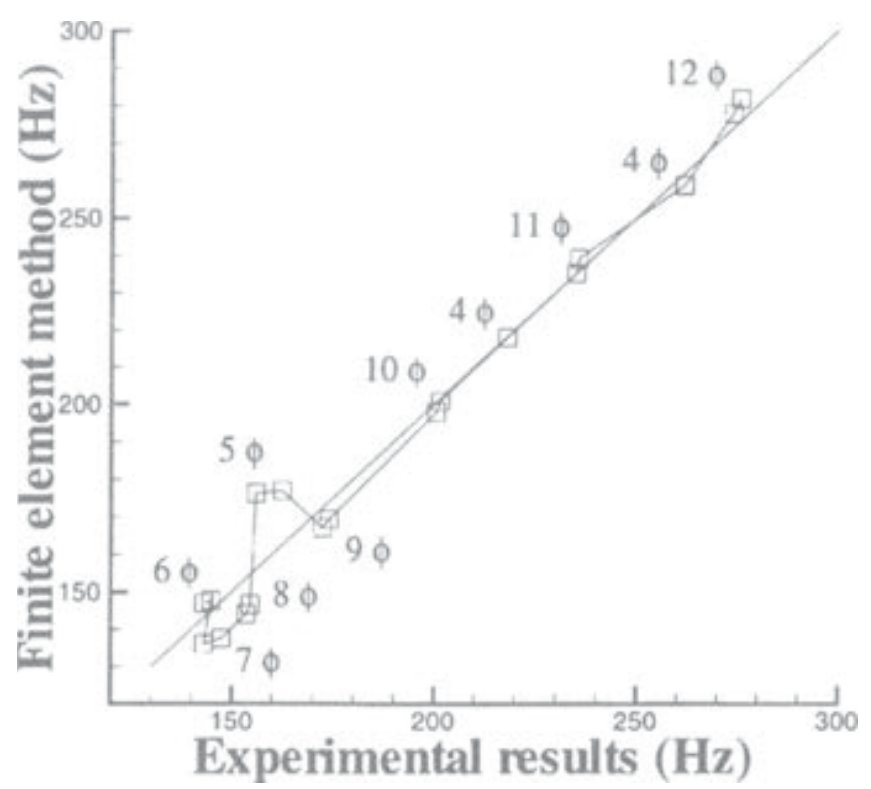

Figure 6. Comparison experimental results - finite element model.

\subsection{CREATING INTERACTION}

The test rig has been used in its current configuration to make first attempts on the interaction phenomenon. The choice of a mode with regular modal shape is more advisable. Moreover, to avoid the high modal density at the beginning of the spectrum, and because of its weak damping, the 10 nodal diameters mode was finally selected. Using the highest rotation frequency of the lathe $(5 \mathrm{~Hz})$, and adapting equation (7) to the situation in which a single blade is implied, the first natural frequency of the blade was set to satisfy the mathematical condition of interaction. The contact was then created on the rotating casing.

The major issue which has to be faced is the lack of symmetry of the casing. The contact is unfortunately not permanent, and the structure is not excited by a constant force. As a result, no significative amplitude in the frequency response is noticeable 
neither on the casing nor on the blade. However, it is interesting to remark that for lower rotation frequency, the 10 nodal diameters mode's answer is higher than the others $(20$ $\mathrm{dB})$. At these rotation speeds, the contact is quite similar to a harmonic excitation. When rotating at $5 \mathrm{~Hz}$, the whole structure experiments impulsions whose effect is the excitation of every mode which is confirmed by the frequency response.

In order to suppress the effect of the geometrical default it is considered to fix an abrasive material on the inner side of the casing and to machine it so as to force the blade/casing contact to be permanent.

\section{Conclusion and Future Prospects}

The interaction phenomena, which have motivated the present work, are of major concern to aircraft designers. The present study is composed of a numerical part dealing with dynamic contact simulations on classical examples, in particular with the Lagrange multiplier method, and an experimental part which consists in identifying the parameters involving modal interaction. The next stage of our work is the simulation and the experimental study of the blade/casing dynamic contact.

\section{References}

1. Belytschko, T., and Neal, M.O.: Contact-impact by the pinball algorithm with penalty and Lagrangian methods, International Journal for Numerical Methods in Engineering 31 (1991), 547-572.

2. Billet, L., and Moreno, J.: Caractérisation des modes de vibration d'une coque de révolution à l'aide d'un vibromètre laser à faisceau tournant, Bulletin SFM, Revue Française de Mécanique 1 (1996), 25-32.

3. Carpenter, N.J., Taylor, R.L., and Katona, M.G.: Lagrange constraints for transient finite element surface contact, International Journal for Numerical Methods in Engineering 32 (1991), 103-128.

4. Chaudhary, A.B., and Bathe, K.J.: A solution method for static and dynamic analysis of threedimensional contact problems with friction, Computers and Structures 24 (1986), 855-873.

5. Staples, B.C.: Excitation of travelling wave response in axi-symmetric structures, Proceeding of the 15th seminar on modal analysis, Lewen, Belgium (19-21 sept 1990),1339-1354.

6. Schmiechen, P., Ewins, D.J., and Bucher, I.: Excitation of arbitrary displacement / velocity conditions in rotationnaly periodic structures, ASME, Design Engineering Technical Conferences 3B (1995), 1353 1360 .

7. Taylor, R.L., and Papadopoulos, P.: On a finite element method for dynamic contact/impact problems, International Journal for Numerical Methods in Engineering 36 (1993), 2133-2140.

8. Tobias, S.A., and Arnold, R.N.: The influence of dynamical inperfections on the vibrations of rotating disks, Proceedings Institute of Mechanical Engineers 171 (1957), 669-690. 